\title{
Variations of the soft tissue thicknesses external to the ribs in Pectus Excavatum patients ${ }^{\text {th }}$
}

\author{
Pedro L. Rodrigues ${ }^{a, b, g, *}$, Bruno Direito-Santos ${ }^{a, b}$, António H.J. Moreira ${ }^{a, b}$, \\ Jaime C. Fonseca ${ }^{c}$, A.C.M. Pinho ${ }^{d}$, Nuno F. Rodrigues ${ }^{g, h}$, Tiago Henriques-Coelho ${ }^{e}$, \\ Jorge Correia-Pinto ${ }^{a, b, f}$, João L. Vilaça ${ }^{a, b, g}$
}

\author{
${ }^{a}$ Life and Health Sciences Research Institute (ICVS), School of Health Sciences, University of Minho, Braga, Portugal \\ ' ICVS/3B's-PT Government Associate Laboratory, Braga/Guimarães, Portugal \\ ${ }^{\mathrm{c}}$ Industrial Electronics Department, University of Minho, Guimarães, Portugal \\ ${ }^{\mathrm{d}}$ Mechanical Department, University of Minho, Guimarães, Portugal \\ e Department of Pediatric Surgery, Hospital São João, Porto, Portugal \\ ${ }^{\mathrm{f}}$ Department of Pediatric Surgery, Hospital de Braga, Portugal \\ ${ }^{\mathrm{g}}$ DIGARC-Polytechnic Institute of Cávado and Ave, Barcelos, Portugal \\ ${ }^{\mathrm{h}}$ HASLab/INESC TEC, University of Minho, Braga, Portugal
}

Received 10 October 2012; revised 2 January 2013; accepted 20 January 2013

\section{Key words: \\ Pectus Excavatum; \\ Nuss procedure; \\ Computed tomography scan; \\ Radiation exposure; Image processing}

\begin{abstract}
Background: Surgical repair of pectus excavatum (PE) has become more popular due to improvements in the minimally invasive Nuss procedure. The pre-surgical assessment of PE patients requires Computerized Tomography (CT), as the malformation characteristics vary from patient to patient.

Objective: This work aims to characterize soft tissue thickness (STT) external to the ribs among PE patients. It also presents a comparative analysis between the anterior chest wall surface before and after surgical correction.

Methods: Through surrounding tissue segmentation in CT data, STT values were calculated at different lines along the thoracic wall, with a reference point in the intersection of coronal and median planes. The comparative analysis between the two 3D anterior chest surfaces sets a surgical correction influence area (SCIA) and a volume of interest (VOI) based on image processing algorithms, 3D surface algorithms, and registration methods.

Results: There are always variations between left and right side STTs $(2.54 \pm 2.05 \mathrm{~mm}$ and $2.95 \pm 2.97 \mathrm{~mm}$ for female and male patients, respectively). STTs are dependent on age, sex, and body mass index of each patient. On female patients, breast tissue induces additional errors in bar manual
\end{abstract}

is Funding: This work was supported by "Fundação para a Ciência e a Tecnologia" (FCT), Portugal, through the R\&D project referenced PTDC/SAU-BEB/ 103368/2008 and the fellowships referenced SFRH/BD/74276/2010, SFRH/BD/68270/2010 and SFRH/BPD/46851/2008.

* Corresponding author. Life and Health Sciences Research Institute (ICVS), School of Health Sciences, University of Minho, $4710-057$ Braga, Portugal. Tel.: +351253604 881; fax: +351253604847.

E-mail addresses: pedrorodrigues@ecsaude.uminho.pt (P.L. Rodrigues), brunodireitosantos@gmail.com (B. Direito-Santos), antoniomoreira@ecsaude.uminho.pt (A.H.J. Moreira), Jaime@dei.uminho.pt (J.C. Fonseca), acmpinho@dem.uminho.pt (A.C.M. Pinho), nfr@ipca.pt (N.F. Rodrigues), henriques.coelho@gmail.com (T. Henriques-Coelho), jcp@ecsaude.uminho.pt (J. Correia-Pinto), joaovilaca@ecsaude.uminho.pt (J.L. Vilaça). 
conception. The distances starting at the deformity's largest depression point at the SCIA are similar in all directions. Some diverging measures and outliers were found, being difficult to find similar characteristics between them, especially in asymmetric patients.

Conclusion: The Nuss procedure metal bar must be modeled according to each patient's special characteristics. The studied relationships between STT and chest surface could represent a step forward to eliminate the CT scan from PE pre-surgical evaluation.

(C) 2013 Elsevier Inc. All rights reserved.

Pectus Excavatum (PE) is characterized by a sternum and costal cartilage depression. It is the most common congenital chest malformation occurring between 1 in 400 and 1 in 1000 births, more frequently in boys than girls in a ratio of 3-5:1 [1].

Despite the fact that patients with PE may occasionally experience symptoms, the reasons for seeking medical assistance are, in most cases, related psychological and social aspects surrounding the body image [2,3]. The minimally invasive surgical correction of PE was described by Dr. Donald Nuss and is characterized by the insertion of a metal pre-bent metal bar through two axillary incisions in the retro-sternal space where it remains in place for two to three years [4]. The anterior chest concavity is evident in patients with the malformation, affecting mainly the lower third of the sternum and ribs than the upper two thirds [5].

The overall assessment of PE patients requires a Computerized Tomography (CT) scan since the depth and symmetry of the malformation vary from patient to patient [6-8]. The radiologic classification of Park et al. [9] allows us to understand the types of malformation and how surgical correction may be difficult in some cases requiring proper bending of the metal bar. However, exposing patients to CT, increases radiation risks $[10,11]$. Regarding the personalized prosthesis, the initial Nuss procedure protocol describes the necessity for manual bar bending but, this is a time wasting procedure often resulting in imperfections that could negatively affect bar adaptation to the patient's chest.

This work aims to characterize soft tissue thickness (STT) external to the ribs among several PE patients as an initial step in developing a non-radiation pre-surgical study. Additionally, it objectively evaluates the anterior chest wall specific modifications after PE surgical repair supporting an individual, pre-surgical and automatic metal bar conception based on 3D images instead of a manual bending.

\section{Methods}

This study is divided into two parts. The first one describes all methods to automatically determine the STTs external to the ribs along the chest wall where the surgical prosthesis (bar) is positioned. These STTs were determined in order to study the relationships between the right and left side of the anterior chest (with respect to the sternum) and taking into account patient age, gender and anatomical indexes. This study enrolled 46 patients submitted to PE surgical correction by the Nuss procedure: 39 males and 7 females. The age at operation ranged from 5 to 18 years (mean of $13.55 \pm 3.42$ ) for male patients and from 12 to 16 years (mean of $14.13 \pm 1.36$ ) for female patients. For both female and male patients, each CT slice size was $512 \times 512$ pixels with a slice thickness of $3 \mathrm{~mm}$. The pixel resolution ranged from 0.50 to $0.73 \mathrm{~mm}$ and 0.46 to $0.84 \mathrm{~mm}$ (for both vertical and horizontal directions) in female and male CT slices, respectively.

The second part describes all methods used to perform a comparative analysis between the anterior chest wall surface before and after PE surgical correction using anterior chest surface $3 \mathrm{D}$ reconstructions superposition in 21 patients. The study was conducted with $\mathrm{CT}$ images acquired at Hospital São João-Porto, Portugal.

\subsection{STT characterization}

Anatomical surgical planes are commonly related to several bone structures, where the prosthesis will be fixed in the PE surgical correction. Therefore, this stage starts with the determination of the $\mathrm{CT}$ axial slice immediately adjacent to the sternum's deepest depression where the surgical prosthetic bar is placed. In most cases, this slice was $15 \mathrm{~mm}$ away from the upper extremity of the xiphoid process. Concerning this image:

1) $\mathrm{PE}$ was quantified and standardized in each patient by comparing numeric indexes among different groups of patients;

2) The STT external to the ribs was calculated through image processing methods and anatomic-surgical references that are related with several bone structures where the prosthesis will be set in the surgical correction.

\subsubsection{PE quantification and standardization}

The following anatomic indexes were manually calculated:

a) Index of Flatness (iF) - ratio between transverse diameter of the thorax (TD, Fig. 1) and anteroposterior distance of left hemithorax (L, Fig. 1): iF $=\mathrm{TD} / \mathrm{L}$;

b) Index of Asymmetry (iA) - ratio between anteroposterior distance of right (R, Fig. 1) and left (L, Fig. 1) hemithorax: $\mathrm{iA}=\mathrm{R} / \mathrm{L}$;

c) Index of Haller $(\mathrm{iH})$ - ratio between transverse diameter of the thorax (TD, Fig. 1) and reduced anteroposterior diameter of the thorax (AP, Fig. 1): $\mathrm{iH}=\mathrm{TD} / \mathrm{AP}$; 


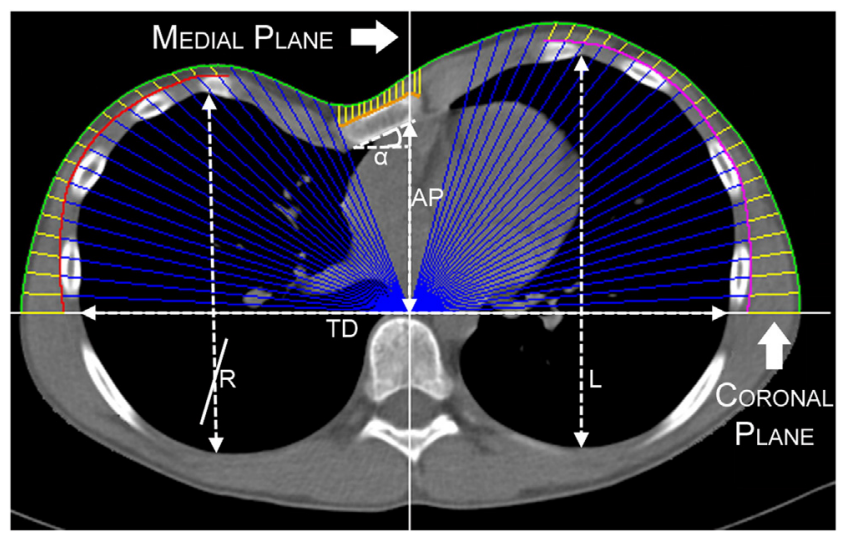

Fig. 1 Representation of different substructures segmented in a CT scan slice: green points are the skin surface; blue lines are used to measure the distance between ribs and skin surface; orange points are sternum external points; red and violet points are the result of piecewise polynomial interpolation corresponding to right and left external ribs points, respectively; yellow lines are the taken measures of STT. The used distances to determine the anatomic indexes are also represented: TD (transverse diameter of the thorax); AP (reduced anteroposterior diameter of the thorax); L (anteroposterior distance of left hemithorax); R (anteroposterior distance of right hemithorax); $\alpha$ (angle of sternum rotation).

d) Index of Depression (iD) - ratio between the anteroposterior distance of left hemithorax (L, Fig. 1) and reduced anteroposterior diameter of the thorax (AP, Fig. 1): iD=L/AP.

The iA is related to the angle of sternal rotation $(\alpha)$, which is a major cause of the costal cartilages' irregular elongation.

\subsubsection{Skin and bone segmentation}

The skin substructure was segmented from the surrounding tissue using an edge detection algorithm based on the image gradient magnitude. All image pixels were vertically and horizontally tracked by recursively selecting all first pixels belonging to image gradient magnitude. The result of this procedure was the skin substructure (skin points anterior to the coronal plane including the mid-axillary line) illustrated in Fig. 1 (green points).

The remaining bone substructures show up with high contrast as the lightest areas (Fig. 1), due to a filter that magnifies all pixels with higher intensity (bone pixels) during CT acquisition time. Therefore, they were segmented by carefully labelling all bone image points as white and all the other points as black using a threshold algorithm. The bone segmentation allows for the determination of the two remaining human planes: coronal and sagittal. The coronal plane was assumed as the one calculated by the vertical component value of the rightmost and leftmost ribs points. The horizontal component of these two points also made possible the calculation of the median plane that vertically bisects the body (right and left side of the patient).
Then, two polynomials were independently created to be suitably associated to the patient's left and right side of the external ribs. The following substructures were created:

1) Right ribs substructure - nearest ribs points to the skin surface, anterior to the coronal plane, including the mid-axillary line and lying in the right limit defined from the median plane (red, Fig. 1);

2) Left ribs substructure - nearest ribs points to the skin surface, anterior to the coronal plane, including the mid-axillary line and lying in the left limit defined from the median plane (violet, Fig. 1);

3) Sternum substructure - sternum points anterior to the coronal plane, including the mid-axillary line and lying between the left and right limits defined from the median plane (orange, Fig. 1).

The purpose of these two polynomials was the determination of STTs external to the ribs where the prosthesis is placed, instead of only considering the places where the ribs exist.

Finally, the STTs external to the ribs were determined through different lines, defined according to the common form of a linear equation. The reference point for all these lines was the intersection of the coronal and median planes, and its slope was incremented from $0^{\circ}$ to $80^{\circ}$, and decremented from $180^{\circ}$ to $100^{\circ}$, on the right and left sides of the patient, respectively. For each line, the STT was determined by the distance between the line point that intersects the ribs spline and the point at the skin surface (yellow, Fig. 1). For the sternum, the vertical distance between the highest points of sternum and the skin surface was calculated.

\subsection{Comparative analysis of the anterior chest wall}

In order to perform a comparative analysis of the anterior chest wall regarding the surgical correction influence area (SCIA) and volume of influence (VOI), this stage reconstructs the anterior thoracic wall according to two different sets of input data, namely $\mathrm{CT}$ imaging (before surgery correction) and 3D scanner (after surgery correction).

Since a pre-surgical CT examination is always carried out, the anterior chest wall (skin points) before surgical correction was acquired through the method described in section 2.1.2. Here, all the 3D CT slices were used instead of considering only the slice of the sternum's greatest depression. The result is shown in the Fig. 2A (grey).

Despite this pre-operative examination, CT scan is not commonly requested after surgical correction. Therefore, the Polhemus laser FastSCAN [12] was used to assess the anterior chest wall points after the prosthesis bar removal. This system enables a computer reconstruction of the anterior thoracic full surface with a laser resolution of $0.5 \mathrm{~mm}$ without any radiation exposure.

In order to achieve a minimal error during acquisition, due to patient and respiratory movements, the system reference was attached to the $10^{\circ}$ left rib of the rib cage. 


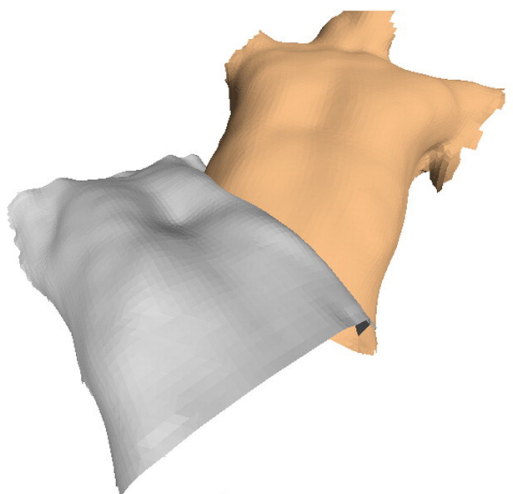

A
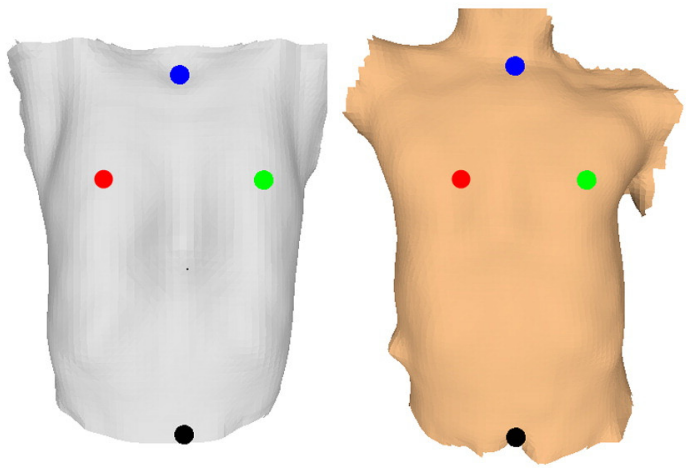

$\mathrm{B}$

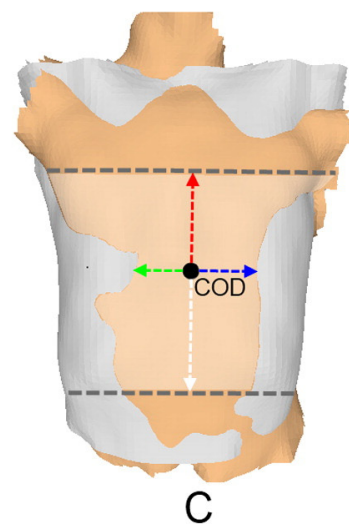

Fig. 2 Overview of the surface reconstruction and registration processes: A) surface reconstruction of the thoracic wall using CT imaging before surgery (grey surface) and the Polhemus Fast Scanner after surgery (orange surface); B) Representation of the registration points to align the two meshes; C) Aligned meshes result and representation of the SCIA illustrating how the measurements were made (arrows representing the directions in which the distances were measured from the COD (center of depression)).

Both point clouds acquired from the 3D scan and CT were used to reconstruct a smooth surface using Radial Basis Functions [13]. The result is shown in Fig. 2A (orange).

In order to evaluate the SCIA and VOI, these two meshes were first aligned using an affine surface registration method [14]. This method determines the translations and/ or rotations in the $3 \mathrm{D}$ space that best align each other. This alignment (Fig. 2C) was achieved by getting four pairs of points from one mesh to the closest distance of the other: one on the neck, one on the navel and one on each breast nipple (Fig. 2B).

Different parameters were defined and analyzed to characterize SCIA, most of them related to the deformity's largest depression point, as follows: upper distance, lower distance (limited at the end of the rib cage), left and right sides. A SCIA 3D study was also performed; the maximum value of each measure was used to define the VOI between the two reconstructions.

\section{Results}

\subsection{STT Study}

Regarding the quantification and standardization of the $\mathrm{PE}$ among all patients, the mean, maximum and minimum values for all anatomic indexes and $\alpha$ were determined and shown in Table 1, for both genders.

Based on Table 1, a primitive classification includes symmetric or asymmetric patients. Besides that, it is possible to distinguish between a well-defined, deep and localized concavity and a wider and shallower concavity. Therefore, it was possible to classify the following groups of various morphologic types of PE:

1) Symmetric patients (27\%) with moderate and severe depressions;

2) Asymmetric patient (73\%) with higher angles $\left(>10^{\circ}\right)$ of sternal rotation. In these patients, the depression centre was not located with sternum centre but was off to right or left side.

The $\mathrm{iH}$ was higher than 3.1 in $61 \%$ and $25 \%$ in male and female patients, respectively. These values show why surgical correction decision based on $\mathrm{iH}$ (equal to 3.25) is not reliable [15].

The STT results, determined through blue lines (Fig. 1) for all female and male patients, are summarized in Fig. 3 and Fig. 4, respectively. The mean, minimum and maximum values of the left and right side of the patients are shown in Table 2. Moreover, it also shows the differences between both sides and the vertical STT between the sternum and skin.

Table 1 Male and female chest numerical indexes.

\begin{tabular}{llllllll}
\hline & & No. patients & $\alpha$ & iD & iH & iA & iF \\
\hline Female & Mean & 7 & $14.03 \pm 12.36$ & $1.87 \pm 0.34$ & $3.02 \pm 0.59$ & $0.99 \pm 0.09$ & $1.62 \pm 0.18$ \\
& Max & & 37.0 & 2.5 & 4.3 & 1.1 & 1.8 \\
\multirow{4}{*}{ Male } & Min & & 0.0 & 1.5 & 2.3 & 0.9 & 1.5 \\
& Mean & 39 & $9.81 \pm 9.42$ & $1.85 \pm 0.31$ & $3.13 \pm 0.70$ & $0.99 \pm 0.08$ & $1.69 \pm 0.18$ \\
& Max & & 36.0 & 2.5 & 4.6 & 1.2 & 0.9 \\
& Min & & 0.0 & 1.3 & 2.0 & 1.4 \\
\hline
\end{tabular}




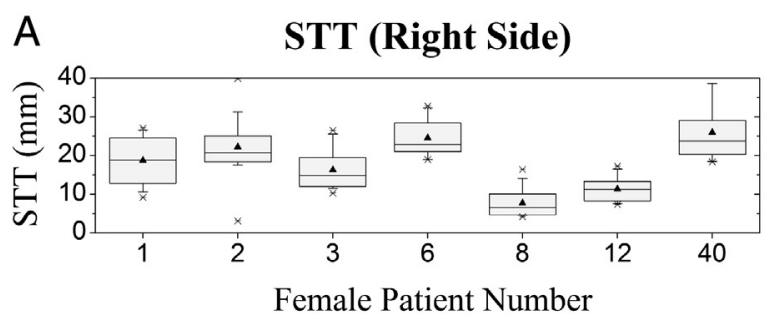

B

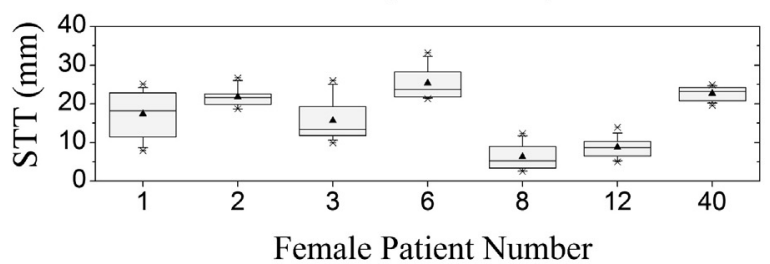

C

STT Difference (Left-Right Side)

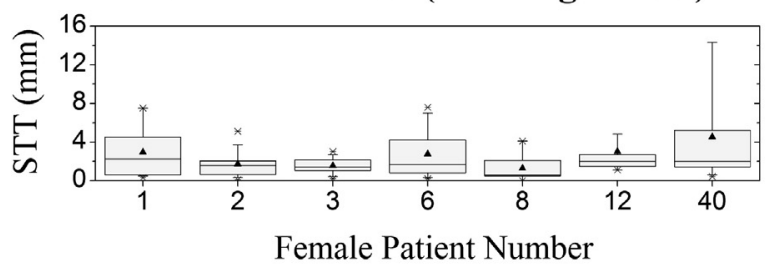

D STT in Different Angles (Right Side)

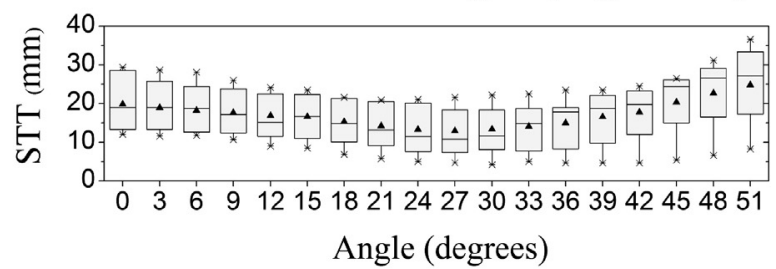

E STT in Different Angles (Left Side)

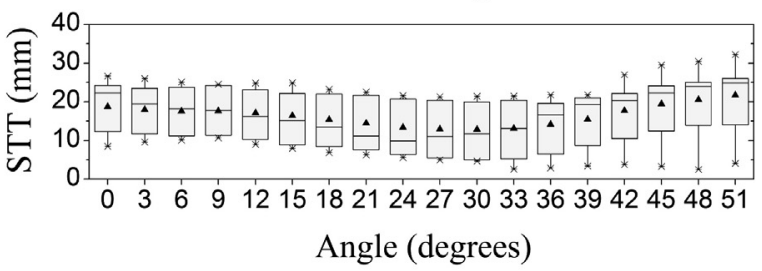

F $\quad$ STT Difference in Different Angles (Left-Right Side)

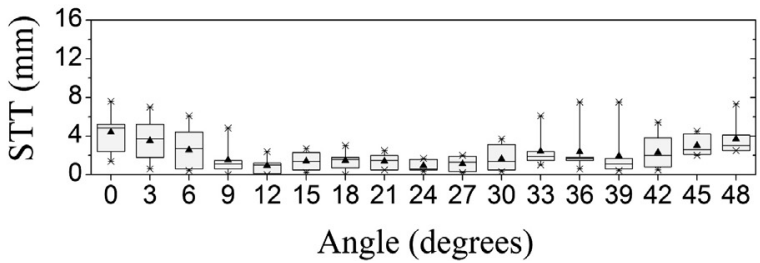

Fig. 3 Boxplots of female results. In A and B are shown STTs for right and left sides of the patients' thoracic wall, respectively; C shows the differences between both sides; D and E show STTs for all female patients along different angles according to the blue lines slope in Fig. 1; F shows STT differences between both sides at each angle of the blue lines.

\subsubsection{Female patients}

Table 2 summarizes the mean, standard deviation, smallest and maximum STT values for female patients' right and left sides.

Fig. 3A and Fig. 3B show the STT variations on the left and right patient's side. The smallest STT value occurred in patient number $8(4.2 \mathrm{~mm}$ and $2.5 \mathrm{~mm}$, for right and left sides, respectively). The highest STT value was obtained in patient number 40 (36.6 mm, right side) and number 6 (32.2 mm, left side).

Fig. 3D and Fig. 3E show the STT variations along the different angles of the thoracic wall (yellow, Fig. 1). The smallest STT occurred when the angle was $30^{\circ}$ and $48^{\circ}$ for right and left sides of the patients, respectively. For both sides, the highest value occurred in $48^{\circ}$ angle; STT values are higher in lower (range from $0^{\circ}$ to $9^{\circ}$ ) and higher (range from $42^{\circ}$ to $48^{\circ}$ ) angles than in middle angles. These higher values are related to more fatty tissues, such as female breasts in higher angles or excess of fat tissue in the lateral female chest. However, some female patients (e.g. patient number 8 and 12, Fig. 3A and Fig. 3B) may not have reached adolescence. This explains small STTs in higher angles since breasts are not yet developed.

The STT's mean value at left breasts was $24.1 \pm 6.03 \mathrm{~mm}$ (range from 15.6 to $32.2 \mathrm{~mm}$ ). At the right breast, the mean of STT was $27.3 \pm 7.24 \mathrm{~mm}$ (range from 18.8 to $36.6 \mathrm{~mm}$ ).
The mean value of fat tissue at lateral female chest was $20.4 \pm 6.58 \mathrm{~mm}$ (range from 10.7 to $30.1 \mathrm{~mm}$ ) and $20.4 \pm$ $7.14 \mathrm{~mm}$ (12.8 to $30.8 \mathrm{~mm}$ ) for left and right patient side, respectively.

As shown in Fig. 3C and Fig. 3F, there are always differences between both sides in all chest wall directions $(2.54 \pm 2.45 \mathrm{~mm})$.

\subsubsection{Male patients}

Table 2 summarizes the mean, standard deviation, smallest and maximum STT values for male patients' right and left sides.

Fig. 4A and Fig. 4B show the STT variations on the patients' left and right side.

On the patients' right side (Fig. 4A), the smallest STT value occurred in patient number $31(2.9 \mathrm{~mm})$ and the highest value occurred in patient number $33(40.0 \mathrm{~mm})$. On the patients' left side (Fig. 4-B), the smallest STT value occurred in patient number $17(2.5 \mathrm{~mm})$ and the highest STT in patient number $33(35.2 \mathrm{~mm})$.

Fig. 4D and Fig. 4E show the STT variations along the different angles of the thoracic wall (yellow, Fig. 1). As illustrated in Fig. 4D and Fig. 4E, the smallest STT occurred when the angle was $24^{\circ}$ and $33^{\circ}$ for patients' right and left sides, respectively. For both sides, the highest value occurred in $0^{\circ}$ angle. 


\section{A STT (Right Side)}

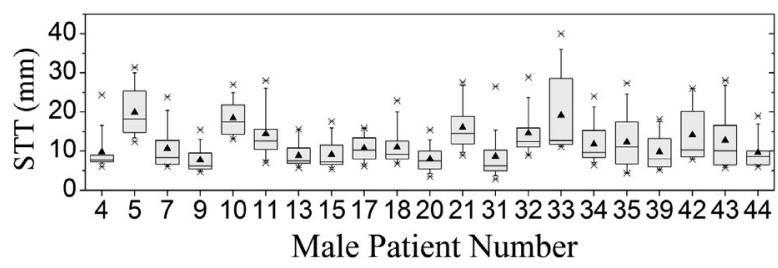

B

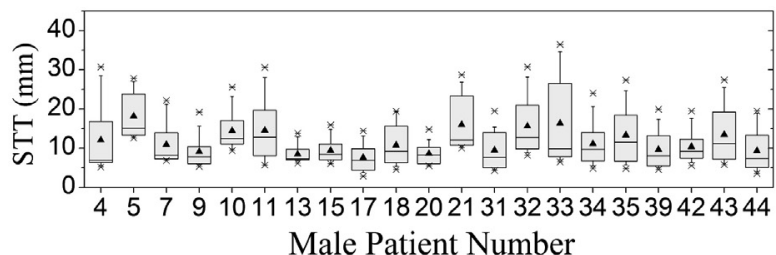

C

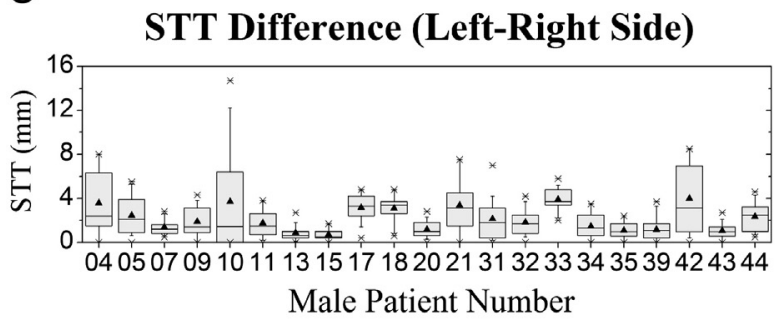

D STT in Different Angles (Right Side)

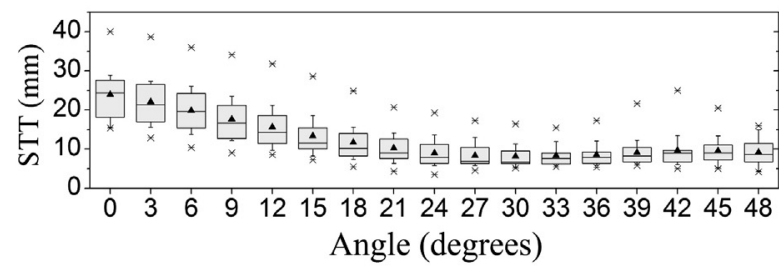

E STT in Different Angles (Left Side)

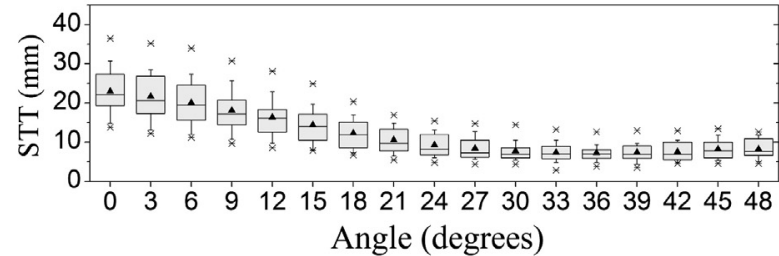

F STT Difference in Different Angles (Left-Right Side)

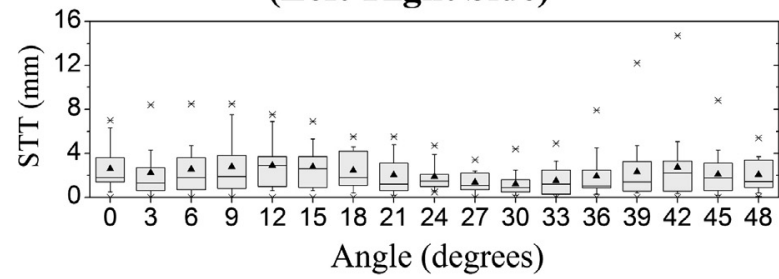

Fig. 4 Boxplots of male results. In A and B are shown STTs for right and left sides of the patients' thoracic wall, respectively; C shows the differences between both sides; D and E show STT for all male patients along different angles according to the blue lines slope in Fig. 1; F shows STT differences between both sides at each angle of the blue lines.

In contrast with female patients, as the angle increases, a decrease of STT value is observed (Fig. 4D and Fig. 4E). Such results are related to breast tissue absence (higher angles).

The STT mean value at left breasts was $9.9 \pm 3.60 \mathrm{~mm}$ (range from 4.1 to $20.9 \mathrm{~mm}$ ). At the right breast, the mean of STT was $11.0 \pm 3.18 \mathrm{~mm}$ (range from 6.1 to $19.3 \mathrm{~mm}$ ). The mean value of the fat tissue in lateral male chest was $23.8 \pm$ $7.11 \mathrm{~mm}$ (range from 15.4 to $36.5 \mathrm{~mm}$ ) and $22.2 \pm 6.23 \mathrm{~mm}$ (12.3 to $36.5 \mathrm{~mm})$ for patients' right and left sides, respectively.

As in the female study, Fig. 4C and Fig. 4F show that there are always differences between both sides in all directions of the chest wall $(2.95 \pm 2.97 \mathrm{~mm})$ in males.

\subsection{SCIA and VOI results}

Following surgical correction of PE, a huge region of the anterior chest wall is reshaped with $3 \mathrm{D}$ transformations above, below, on the left and on the right sides of the deformity's largest depression point. Fig. 5A and Fig. 5B show that the mean values of upper and lower distances, as well the distances on the right and left side, are similar. This means that surgical correction of PE usually occurs similarly in all directions of the anterior chest wall, starting at the point of greatest depression. However, some diverging measures and outliers were also found revealing the difficulty in finding similar characteristics between them, especially in asymmetric patients. Equal results relation was noticed at SCIA and VOI analysis.

Table 2 Summary of STT differences.

\begin{tabular}{llllll}
\hline & Right & Left & Left-Right & Sternum-Skin \\
\hline Female & Mean & $17.11 \pm 7.13$ & $16.46 \pm 7.37$ & $2.54 \pm 2.45$ & $11.26 \pm 6.30$ \\
& Max & 36.6 & 32.2 & 17.3 & 2.34 \\
\multirow{3}{*}{ Male } & Min & 4.2 & 2.5 & 0.0 & 25.00 \\
& Mean & $12.26 \pm 6.74$ & $11.94 \pm 6.93$ & $2.95 \pm 2.97$ & $6.17 \pm 3.70$ \\
& Max & 40.0 & 35.2 & 15.0 & 2.57 \\
& Min & 2.9 & 2.5 & 0.0 & 25.57 \\
\hline
\end{tabular}



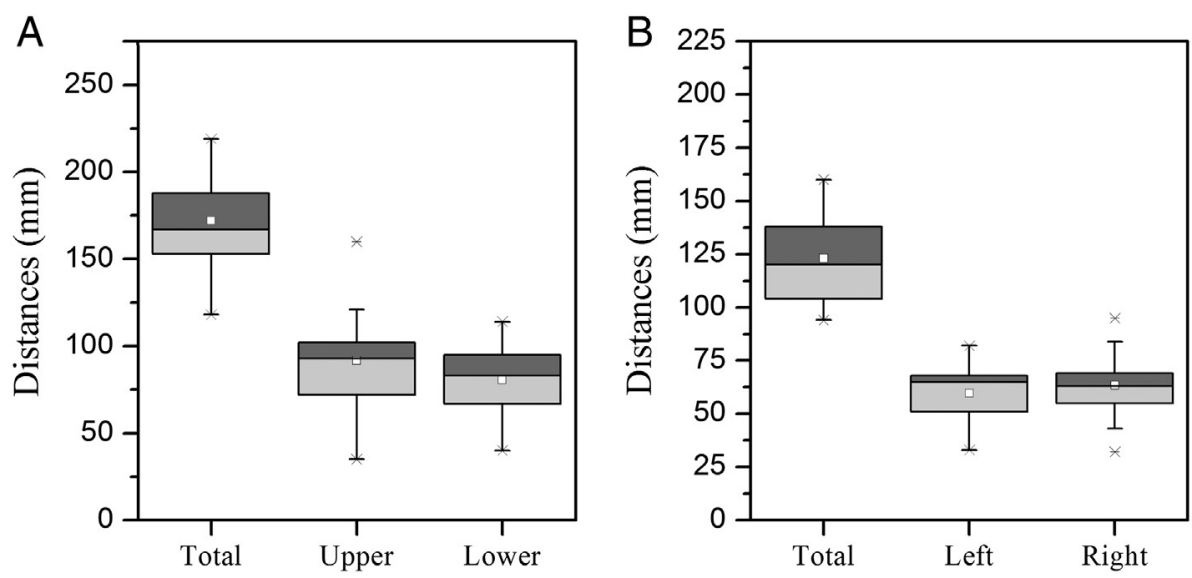

Fig. 5 Measurement results of the PE surgical correction outcome along the coronal plan: A — boxplot of the vertical distances; B boxplot of the horizontal distances.

The second group of measurement parameters performs a tridimensional analysis in each patient SCIA. Fig. 6A displays the full range of distances variation between the two meshes (before and after surgical correction) at the center of depression (mean value of $12 \pm 8.7 \mathrm{~mm}$ ). For high deformities this value could increase significantly, up to $35 \mathrm{~mm}$.

SCIA changes along the coronal plan, where $75 \%$ of the patients present a SCIA between $10.0 \times 10^{3} \mathrm{~mm}^{2}$ and $31.9 \times 10^{3} \mathrm{~mm}^{2}$ (Fig. 6B) (mean value of $21.5 \times 10^{3} \pm$ $9.1 \times 10^{3} \mathrm{~mm}^{2}$ ). The volume of the SCIA between the two anterior chest wall surface reconstructions indicates a mean volume of $152 \times 10^{3} \mathrm{~mm}^{3}\left( \pm 94.5 \times 10^{3} \mathrm{~mm}^{3}\right)$ (Fig. $6 \mathrm{C}$ ).

\section{Discussion}

PE surgical repair has become more frequently performed due to improvements in the minimally invasive Nuss procedure during the last twenty years [16]. This technique is the standard procedure adopted by most surgeons because of the marked advantages over the Ravitch technique $[6,17]$. Over the years, there have been many modifications in the Nuss procedure protocol in order to provide greater security and better post-operative outcomes [16]. Through our clinical practice, it was noticed that the post-surgical aesthetic results do not always meet initial expectations. This is a major problem since the whole process is aimed at correcting a malformation that may adversely affect the emotional and psychological status of the patient. Thus, although the surgical correction is carried out with a minimally invasive technique with diminished risk for the patient, there is a long way to go before obtaining perfect surgical results.

In order to understand which variables could be affecting the performance of the surgical correction, this work presents a study of the chest modifications after metal bar placement. It was noticed that surgical correction usually occurs similarly in all directions of the anterior chest wall starting at the point of greatest depression. However, the diverging measures and outliers found associated with different degrees of malformation severity reveal the need to adjust the metal bar to the specific characteristics of the malformation. These results confirm objectively the initial premise postulated by Park et al. [9]. In order to overcome the shortcomings of the Nuss technique Vilaça et al. [18] developed a system for automatically modelling and bending the metal bar, allowing its pre-operative personalization according to the chest morphology of each patient. The size and shape of the prosthesis are determined using the ribs
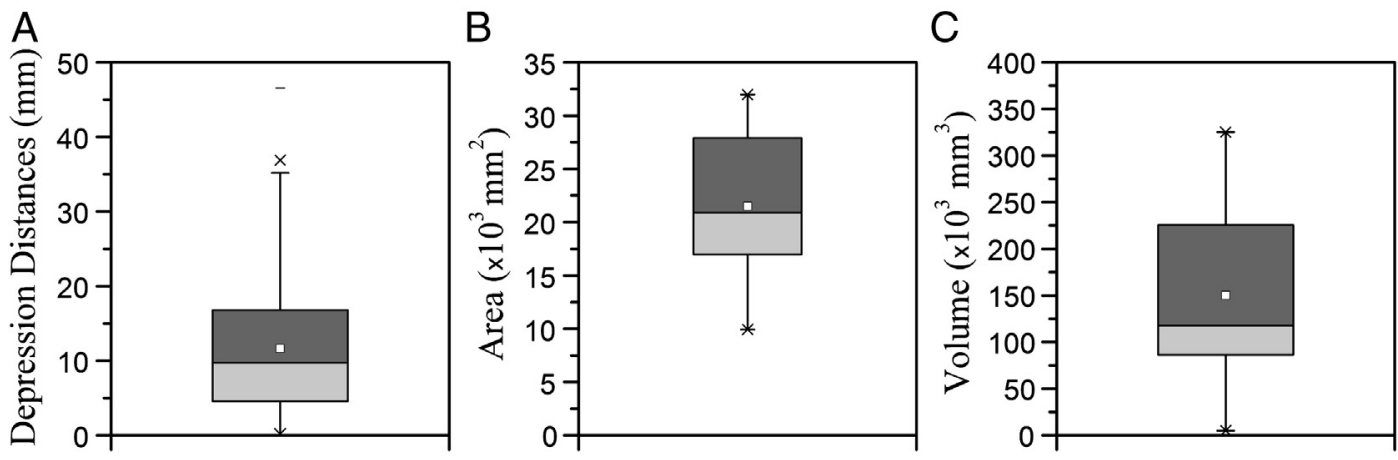

Fig. 6 Tridimensional analysis of the SCIA. 
dimensions and position acquired by CT scan. This system was already tested successfully in more than 40 patients at Hospital S Joao-Porto, Portugal.

The asymmetry and other specific features of the malformation in a given individual are easily studied by CT images making it possible to define the necessary maneuvers for an appropriate correction of the deformity $[1,19]$. Despite CT being an examination routinely ordered in the preoperative evaluation of the patient and used in Vilaça et al. system [18], its use may be questionable since the benefit-risk ratio is not known. CT scan produces larger amounts of radiation compared with other imaging modalities. Without a combination of sufficient technical understanding and appropriate application, this technology can increase the risk and the probability of cancer, hair loss and dermatitis with cumulative doses (radiation) [10,20-24]. Most PE patients seek healthcare advice during late childhood and adolescence when they are most affected by social and psychological commitments [2,3]. These age groups are inherently more radiosensitive, have the potential to be exposed to more radiation during their lifetimes and have more years of remaining life, where a radiation-induced cancer could develop $[10,11]$. Thus, it is predictable that CT scan will be abandoned as a compulsory diagnostic study in the future as new image modalities without inherent danger to the patient are adopted.

STT characterization among PE patients noticed that regardless of malformation symmetry, there are always variations between the left and right sides' STT. Thus, in addition to malformation symmetry, the bar should be modelled and bent according to position and dimension of the ribs. Using a template that only copies the chest wall curvature can require intraoperative readjustments as the distance between skin and ribs differs with patient's side as well as with the position along the thoracic cage. Moreover, STTs are also dependent on age, sex, and body mass index of each patient. In female patients, breast tissue could induce additional errors in manual bar preparation.

This study is a first step in a project that aims to replace the CT scan with a 3D scanner for establishing the preoperative diagnosis. Nowadays, there has been no insight on $\mathrm{PE}$ types and consequently there is no development of specific approaches for special characteristics of the malformation. A 3D laser scanner is a device that analyses the position and orientation of a real-world object, enabling the computer to reconstruct its full 3D surface. Thus, a 3D scanner will be used to create patient skin surface information without any danger to the patient. Since the prosthetic bar should be modelled with bone structure position and the 3D scanner only acquires skin surface, the STT relationships will be the input to soft computing techniques, such as artificial neural networks (ANNs) to indirectly calculate the position of the ribs. ANNs are mathematical or computational models that change their structures based on external or internal information that flows through the network during a learning process. In a future work, an ANN will be developed/trained to estimate rib position though skin surface information with an accuracy to characterize the PE severity and potential to provide information to automatically model/bend the chest prosthesis bar using the system described by Vilaca et al. [18].

\section{Conclusion}

The STTs external to the ribs study presented here show that symmetric or asymmetric patients always have asymmetric variations in STT by comparing both sides of the patients' chest. SCIA and VOI characterization found diverging measures and outliers indicating the difficulty in finding similar characteristics between them, especially in asymmetric patients. Both studies highlight that in the Nuss procedure the metal bar must be modeled according to each patient's special characteristics using ribs position and dimension. Metal bar modelling should take into account the asymmetry of each patient, instead of using a template that only copies the chest wall curvature.

In order to document individual characteristics without the use of a CT scan, which potentially submits the patient to higher radiation exposure, a new imaging method is proposed. Further work is needed to develop ANNs to determine the ribs position with an accuracy to characterize the severity of the PE defect and to provide information to automatically model/bend the chest metal bar.

\section{Acknowledgments}

The authors of this work would like to thank the Department of Pediatric Surgery of Hospital de S. João of Porto.

\section{References}

[1] Fokin AA, Steuerwald NM, Ahrens WA, et al. Anatomical, histologic, and genetic characteristics of congenital chest wall deformities. Semin Thorac Cardiovasc Surg 2009;21:44-57.

[2] Koumbourlis AC. Pectus excavatum: pathophysiology and clinical characteristics. Paediatr Resp Rev 2009;10:3-6.

[3] Kelly Jr RE, Cash TF, Shamberger RC, et al. Surgical repair of pectus excavatum markedly improves body image and perceived ability for physical activity: multicenter study. Pediatrics 2008;122: 1218-22.

[4] Donald N, Robert EK, Daniel PC, et al. A 10-year review of a minimally invasive technique for the correction of pectus excavatum. J Pediatr Surg 1998;33:545-52.

[5] Hebra A. Minimally invasive repair of pectus excavatum. SeminThorac Cardiovasc Surg 2009;21:76-84.

[6] Kelly Jr RE. Pectus excavatum: historical background, clinical picture, preoperative evaluation and criteria for operation. Semin Pediatr Surg 2008;17:181-93. 
[7] Park HJ, Lee SY, Lee CS. Complications associated with the Nuss procedure: analysis of risk factors and suggested measures for prevention of complications. J Pediatr Surg 2004;39:391-5 [discussion 91-5].

[8] Jaroszewski D, Notrica D, McMahon L, et al. Current management of pectus excavatum: a review and update of therapy and treatment recommendations. J Am Brd Fam Med: JABFM 2010;23: 230-9.

[9] Park HJ, Lee SY, Lee CS, et al. The Nuss procedure for pectus excavatum: evolution of techniques and early results on 322 patients. Ann Thorac Surg 2004;77:289-95.

[10] Paterson A, Frush DP, Donnelly LF. Helical CT of the body: are settings adjusted for pediatric patients? AJR Am J Roentgenol 2001;176:297-301.

[11] Hollingsworth C, Frush DP, Cross M, et al. Helical CT of the body: a survey of techniques used for pediatric patients. AJR Am J Roentgenol 2003;180:401-6.

[12] Polhemus. FastSCAN. http://www.polhemus.com/?page=Scanning Fastscan (04-01 2011, date last accessed).

[13] Savchenko VV, Pasko AA, Okunev OG, et al. Function representation of solids reconstructed from scattered surface points and contours. Comput Graph Forum 1995;14:181-8.

[14] Aiger D, Mitra NJ, Cohen-Or D. 4-points congruent sets for robust pairwise surface registration. Acm T Graphic 2008;27.
[15] St Peter SD, Juang D, Garey CL, et al. A novel measure for pectus excavatum: the correction index. J Pediatr Surg 2011;46:2270-3.

[16] Nuss D. Minimally invasive surgical repair of pectus excavatum. Semin Pediatr Surg 2008;17:209-17.

[17] Pilegaard HK, Licht PB. Early results following the Nuss operation for pectus excavatum - a single-institution experience of 383 patients. Interact Cardiovascular Thorac Surg 2008;7:54-7.

[18] Vilaça J, Pinho A, Correia-Pinto J, et al. System for automatic and personalized modelling/bending of surgical prosthesis for correction of pectus excavatum based on pre-surgical imagiology information, Patent: WO2009/035358. 2009.

[19] Krasopoulos G, Goldstraw P. Minimally invasive repair of pectus excavatum deformity. Eur J Cardiothorac Surg 2011;39:149-58.

[20] Donnelly LF, Frush DP. Pediatric multidetector body CT. Radiol Clin North Am 2003;41:637-55.

[21] Frush DP, Donnelly LF. Helical CT in children: technical considerations and body applications. Radiology 1998;209:37-48.

[22] Paterson A, Donnelly LF, Frush DP. The pros and cons of imaging options. Contemp Pediatr 2001;18:p73.

[23] Smith-Bindman R. Is computed tomography safe? New Engl J Med 2010;363:1-4.

[24] Pearce MS, Salotti JA, Little MP, et al. Radiation exposure from CT scans in childhood and subsequent risk of leukaemia and brain tumours: a retrospective cohort study. Lancet 2012. 